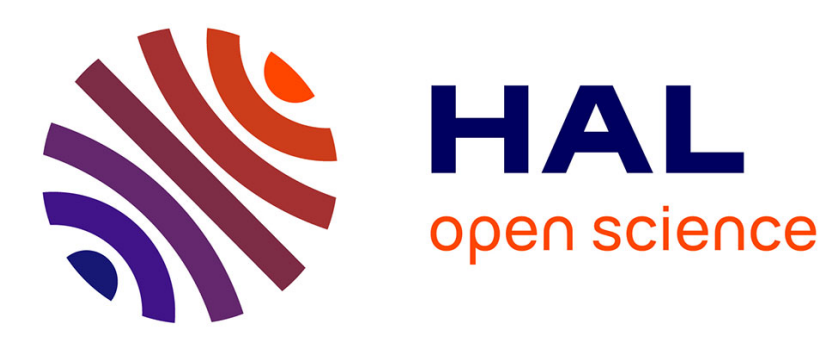

\title{
Les troubles du sommeil du bébé et du jeune enfant : revue de la littérature et analyse psychodynamique
}

\author{
Fleur Breil, Ouriel Rosenblum, Annick Le Nestour
}

\section{To cite this version:}

Fleur Breil, Ouriel Rosenblum, Annick Le Nestour. Les troubles du sommeil du bébé et du jeune enfant: revue de la littérature et analyse psychodynamique. Devenir - Revue européenne du développement de l'enfant, 2010, 22 (2), pp.133 - 162. 10.3917/dev.102.0133 . hal-01516964

\section{HAL Id: hal-01516964 \\ https://hal.science/hal-01516964}

Submitted on 26 Jun 2017

HAL is a multi-disciplinary open access archive for the deposit and dissemination of scientific research documents, whether they are published or not. The documents may come from teaching and research institutions in France or abroad, or from public or private research centers.
L'archive ouverte pluridisciplinaire HAL, est destinée au dépôt et à la diffusion de documents scientifiques de niveau recherche, publiés ou non, émanant des établissements d'enseignement et de recherche français ou étrangers, des laboratoires publics ou privés. 


\section{Clinique}

\section{Les troubles du sommeil du bébé et du jeune enfant: revue de la littérature et analyse psychodynamique}

\section{Sleep disorders in infancy and childhood: review of the literature and psychodynamic analysis}

Fleur Breil ${ }^{1}$, Ouriel Rosenblum ${ }^{2}$ et Annick Le Nestour ${ }^{3}$

\section{Introduction}

Le sommeil est une fonction vitale. Il occupe plus de la moitié du temps de la première année de vie, à peu près le tiers ensuite chez l'adulte. Le sommeil du bébé, au-delà de son développement neurophysiologique, est intimement lié aux aspects de la vie psychologique. Ainsi, on ne peut isoler l'étude du sommeil de ce qui se passe pendant la veille: "Vigilance et sommeil sont les deux aspects d'une même fonction» (A. Doumic [33]). S'endormir impose le lâcher prise. C'est accepter de laisser son corps sans contrôle et aussi de perdre quelque chose du plaisir et du temps. «Tomber de sommeil» peut représenter une solution plus radicale quand ce travail de désinvestissement apparaît trop coûteux. Dans ce moment de transition, pour accompagner le bébé dans ce retrait temporaire du monde environnant, les parents vont être les régulateurs de l'expérience du laisser-aller et du lâcher prise. Le sommeil du bébé s'organise à l'intérieur de la relation parent-enfant. Les parents vont prêter leur «capacité de rêverie» (W.R. Bion [19]) au bébé, afin qu'il puisse s’éloigner de sensations ou de pensées trop excitantes. Ils sont les «gardiens du sommeil» de l'enfant (J. McDougall [90]) jusqu'à ce que le rêve assure cette fonction de «gardien du sommeil» (S. Freud [47]).

Le sommeil est un indicateur parmi les plus sensibles de l'équilibre du nourrisson à la construction duquel il participe. Si toute cause organique est écartée, les troubles du sommeil s'inscrivent dans les désordres psychosomatiques du premier âge, où l'accent est mis sur la qualité de la relation mère-père-enfant. Par leur fréquence et leur retentissement sur

\footnotetext{
${ }^{1}$ Assistante spécialiste, Hôpital Robert Ballanger, Service du Dr Berdah, Aulnay sous Bois (93).

${ }^{2}$ Professeur de psychologie clinique et psychopathologie, Université de Bourgogne, Dijon.

${ }^{3}$ Pédopsychiatre, chef de service VII Intersecteur des Hauts de Seine, L'Aubier, Bourg-la-Reine, EPS Erasme.
} 
la vie familiale, les troubles du sommeil se trouvent au centre de la clinique fonctionnelle de la petite enfance. Le père est toujours directement impliqué, plus souvent que dans d'autres troubles [76]. Les troubles du sommeil du bébé attaquent le corps des parents qui ne dorment pas et ne peuvent donc plus se ressourcer dans leurs rêves.

Depuis plusieurs années, les professionnels de la petite enfance constatent une recrudescence des troubles du sommeil chez des enfants de plus en plus jeunes. Devenant un problème de santé publique dans les sociétés occidentales, ces troubles mobilisent les chercheurs de différents pays pour en étudier la genèse, le traitement, et la prévention, de manière à y remédier. La physiologie du sommeil, étudiée dans les conditions usuelles, et non plus en laboratoire, ainsi que la découverte de la précocité des compétences relationnelles, de l'organisation et des rythmes de la relation mère-enfant, constituent deux apports majeurs à la compréhension des troubles du sommeil du bébé [60]. Les troubles du sommeil représentent une clinique d'une grande diversité et s'expriment différemment, en fonction de l'âge, du stade de développement traversé, et du moment de leur survenue au cours du cycle de sommeil.

Après un rappel sur l'ontogenèse du sommeil, nous proposons une revue de la littérature sur les données épidémiologiques et cliniques des troubles durant les deux premières années de vie de l'enfant. Notre objectif est, ensuite, de développer les principaux axes étiopathogéniques impliqués, et, enfin, les principes thérapeutiques, à cette phase précoce du développement.

\section{Rappel sur l'ontogenèse du sommeil}

Le sommeil se construit et s'organise dès la période fotale [7, 26, 27, 35, $38,39,76,77,89]$. Ses caractéristiques évoluent très rapidement lors des premiers mois de vie. En effet, les composantes qui caractérisent le sommeil de l'adulte se mettent en place dans les deux premières années. A la naissance, le nouveau-né perd l'organisation circadienne du sommeil de la vie fotale pour adopter un sommeil polyphasique: il dort 15 à 17 heures par jour par périodes de 3-4 heures réparties sur l'ensemble du nycthémère; on parle de rythme ultradien de sommeil; ce rythme correspond à l'alternance de cinq stades de vigilance bien déterminés: le sommeil calme (équivalent du sommeil lent profond de l'adulte), le sommeil agité (équivalent du sommeil paradoxal), l'état de veille calme au cours duquel le nouveau-né peut, dès les premiers jours de vie, être attentif à son envi- 
ronnement, et les états de veille agitée sans ou avec pleurs. C'est entre le $1^{\text {er }}$ et le $3^{\mathrm{e}}$ mois que le sommeil va se transformer de la façon la plus importante avec l'apparition d'une périodicité jour/nuit, l'apparition des rythmes circadiens biologiques, avec notamment la sécrétion rythmique de la mélatonine et enfin, la maturation électroencéphalographique du sommeil avec la mise en place du sommeil paradoxal et des différents stades du sommeil lent qui caractérisent le sommeil de l'adulte. A partir de 3 mois, les états de vigilance s'organisent sur un rythme circadien de 24 heures. Dès l'âge de 9 mois, la structure du sommeil nocturne ressemble à celle de l'adulte. Même les bébés qui «font leur nuit» se réveillent au cours de la nuit. Ces éveils physiologiques surviennent à chaque changement de cycle; leur nombre va progressivement diminuer, de même que la durée du sommeil, au fil des années.

La connaissance de cette ontogenèse du sommeil est indispensable pour identifier les perturbations « authentiques» du sommeil et les différencier des caractéristiques normales d'un sommeil en pleine maturation. La pathologie du sommeil du bébé doit être évaluée en fonction des caractéristiques du sommeil selon le stade du développement traversé et les particularités individuelles car, dès la naissance, il y a des grands et des petits dormeurs [76, 77]. En effet, la variabilité interindividuelle concernant les durées de sommeil et leur répartition sur le nycthémère se définit dès les premières semaines de vie. Ces variations peuvent être attribuées à des facteurs environnementaux, dont le rôle sur le sommeil est connu. L'implication de facteurs génétiques polymorphes est maintenant reconnue [18]. Ainsi, les mutations et polymorphismes de certains gènes sont la cause de phénotypes pathologiques, tandis que d'autres contribuent à des variations interindividuelles dans les différents aspects du sommeil. Cependant ces facteurs génétiques et environnementaux n'expliquent pas toute la variabilité observée. Chaque bébé module ses passages par ses états de veille et de sommeil dans la rencontre entre ses caractères individuels et son milieu dyadique et triadique. La pathologie du sommeil du bébé s'organise dans la sphère des interactions précoces entre le bébé, la mère et le père.

\section{Epidémiologie}

\section{Prévalence}

Les troubles du sommeil du bébé et du jeune enfant représentent un problème de santé publique dans nos pays occidentaux $[6,7,8,9,10,12,26,27$, $84,99,112]$. Les chiffres de prévalence varient entre 20 et $50 \%$ en fonction 
des définitions du trouble utilisées et des différences dans les échantillons d'enfants étudiés.

L'estimation de ce trouble fonctionnel est difficile. Les définitions utilisées sont différentes selon les études et manquent parfois de cohérence entre elles, même si chacune intègre des critères de fréquence, de sévérité et de chronicité. La plupart des études ne distinguent pas opposition au coucher, troubles de l'endormissement et réveils nocturnes, conduisant à des chiffres de prévalence globaux [96]. La définition des troubles chez le bébé et le jeune enfant est plus complexe que chez l'adulte. Elle nécessite notamment de prendre en compte la dimension développementale. Des troubles du sommeil transitoires peuvent se comprendre dans un contexte d'évolution physique, émotionnelle et cognitive aux différents stades de développement [96]. De plus, pour certains auteurs, les sources d'information basées sur les descriptions parentales sont peu fiables, considérées comme trop subjectives [95, 116]. En effet, un grand nombre de variables interviennent comme le « seuil de tolérance familiale» [124], concept qui a été particulièrement développé par les chercheurs épidémiologistes transculturels, mais aussi l' impact du trouble dans la vie familiale [124], et bien sûr l'existence d'une psychopathologie parentale [96]. Ainsi, certaines études ont recours à des méthodes d'évaluation objectives du trouble qui complètent les informations recueillies auprès des parents. L'enregistrement vidéoscopique et l'utilisation d'un actigraphe sont les deux techniques utilisées en ambulatoire pour mesurer les perturbations du sommeil du jeune enfant [116, 121].

Les études s'accordent sur la fréquence croissante des troubles du sommeil du jeune enfant dans nos sociétés occidentales. L'importance accordée au monde du travail, aux dépens des conditions de vie de l'enfant [77], ou encore le malaise des jeunes mères dans nos sociétés actuelles, souvent seules, stressées, peu aidées, désemparées du fait de l'éclatement des familles et contraintes à des rôles sociaux [31,76, 89], sont mises en avant. Les auteurs des études transculturelles [23, 55, 56, 124], insistent sur la participation des représentations contemporaines du sommeil du jeune enfant, qui sous-tendent des pratiques de maternage visant à éloigner l'enfant de l'adulte.

\section{Sexe Ratio}

Les résultats des études concernant la répartition des troubles du sommeil en fonction du genre apparaissent homogènes [59, 99, 113, 11, 29]. La majorité d'entre elles concluent à une distribution égale dans les deux sexes. 


\section{Age d'apparition}

Deux phases électives dans l'éclosion des troubles [33, 77, 89] sont identifiées: d'une part la fin du premier trimestre, d'autre part la seconde année de vie. La plupart des insomnies du nourrisson s'installent dans un délai de quelques semaines. La phase d'éveil, au $2^{\mathrm{e}}$ ou $3^{\mathrm{e}}$ mois est une période vulnérable, au cours de laquelle l'enfant est volontiers «criard, excitable et nerveux», mais commence aussi à sourire électivement aux visages humains; c'est une période pendant laquelle les soins maternels sont moins exclusifs et où apparaissent les premières difficultés relationnelles. Les troubles du sommeil surgissent avec une grande fréquence dès le début de la deuxième année. Beaucoup d'insomnies de cette période font partie des variations de la normale [77, 89].

Depuis plusieurs années, des perturbations du sommeil de plus en plus sévères et survenant chez des enfants de plus en plus jeunes sont rapportées [59, 60, 76, 77, 82, 83]. Certaines insomnies sont décrites comme survenant « dès la naissance», ou presque, « dans les toutes premières semaines» [89]. Cependant, l'âge à partir duquel le clinicien est en mesure de pouvoir authentifier un «trouble du sommeil» reste controversé. Pour certains auteurs, il n'existe pas de trouble du sommeil dans les premiers mois de vie car il n'existe pas de rythme circadien. Autrement dit, on n'a pas le droit de parler de perturbation du sommeil avant que se mettent en place les rythmes veille/sommeil stables de 1'enfant, c'est-à-dire avant 2-3 mois [38], voire 6 mois [84]. Pour d'autres auteurs [59, 60], il ne faut pas «banaliser ces insomnies très précoces sous le couvert des réveils normaux de cet âge». Moins fréquentes dans la pratique clinique, elles doivent attirer l'attention du clinicien et nécessitent une investigation approfondie.

\section{Anamnèse obstétricale}

L'importance des évènements périnataux dans la survenue des troubles est connue $[13,20,25,28,51,59,63,91,92,93,94,108,117,126]$.

Certains facteurs liés à la grossesse sont rapportés. Certains auteurs [13] suggèrent que les troubles du sommeil de la femme enceinte pourraient retarder le développement des patterns de sommeil de l'enfant et conduire à une phase plus longue de réveils nocturnes et d'autres problèmes de sommeil. La corrélation entre les perturbations psychiatriques, notamment thymiques, dans lesquelles les troubles du sommeil de la gestante peuvent s'intégrer, et la survenue de troubles du sommeil chez le bébé, est maintenant bien admise. En effet, de nombreuses études [13, 20, 51, 59, 63, 102] ont établi un lien entre la présence d'un haut niveau d'angoisse et de 
dépression maternelle pendant la grossesse et les troubles du sommeil de l'enfant. La primiparité [117] apparaît être un facteur de risque. Les intoxications périnatales (alcool, tabac, médicament, drogue, etc.) [108], avec notamment le syndrome d'alcoolisation fotale, sont responsables de syndrome de sevrage qui s'accompagnent de perturbations majeures des cycles de sommeil pendant des mois, voire des années.

Les mêmes études retrouvent un effet significatif des caractéristiques de l'accouchement sur le développement du sommeil pendant les premiers jours de vie (enfants nés par césarienne, accouchement par le siège et naissances multiples [28]).

Enfin, les facteurs liés aux caractéristiques du nouveau-né sont bien connus, mais les résultats des études parfois contradictoires. La prématurité constitue un facteur de risque bien connu [108]. Les pathologies postnatales sont dominées par le reflux gastro-œsophagien qui prend une part majeure dans les troubles du comportement et du sommeil du prématuré. De même, les septicémies, certains retards de croissance intra-utérins (hypotrophie fotale), l'anoxie néonatale, les bronchodysplasies sont impliqués. Les troubles du sommeil qui revêtent souvent l'allure de l'insomnie tapageuse et agitée, font partie des perturbations fréquemment rencontrées dans la première enfance des enfants nés prématurés [77]. Ils sont alors rarement isolés mais s'intègrent dans un tableau associant d'autres troubles (anorexie, spasme du sanglot, activités rythmiques, etc.). T. Moore et L.E. Ucko [99] proposent une association entre l'anoxie néonatale et la survenue de troubles du sommeil associés à une «hyperactivité» chez le bébé. Enfin, il a été montré que l'exposition du jeune enfant au tabac et à l'alcool dans le lait maternel a des conséquences à court terme sur ses patterns veille/sommeil [91,92,93,94].

\section{Milieu familial}

Tous les niveaux socio-économiques sont représentés [59, 110]. Les résultats des études concernant l'influence du rang dans la fratrie et la position d'enfant unique dans la survenue du trouble sont contradictoires. De nombreuses études $[59,84,112,113]$ ne retrouvent pas de différence significative entre réveilleurs et non réveilleurs quant à l'ordre des naissances. Cependant, quelques études suggèrent que ceux qui se réveillent sont, plus souvent, des premiers-nés. Dans l'étude de A. Pelissolo, et al. [110], les enfants sont, le plus souvent, les aînés de la fratrie ou des enfants uniques. La co-occurrence du trouble chez plusieurs enfants issus de la même fratrie est intéressante [123]. 
Les études s'accordent sur la fréquence élevée durant la grossesse d'évènements majeurs de préoccupations pour la gestante $[59,77,112]$ : conflit ou rupture du couple parental, perte d'emploi, deuil dans la famille ou dans l'entourage, tensions intrafamiliales, etc., ainsi que sur la fréquence d'un diagnostic psychiatrique chezle «caregiver». En effet, depuis longtemps, les problèmes de sommeil des jeunes enfants sont mis en lien avec les problèmes psychiatriques de leurs parents. De nombreuses études s'accordent sur l'association entre dépression maternelle et troubles du sommeil du bébé $[14,32,59,63,74,75,79,84,111,123,138]$. Desfacteurs génétiques et psychosociaux sont invoqués, mais des caractéristiques interactives spécifiques sont maintenant bien décrites [32, 59]. Ces troubles ont également été reliés à un modèle d'attachement insécure des mères, ainsi qu'aux angoisses maternelles. Dans l'étude de D. Benoît [16], 100\% des mères d'enfants souffrant de troubles du sommeil ont un attachement insécure, évalué par 1'Adult Attachment Interview [85], comparées à 57\% des mères dans le groupe contrôle. Certaines catamnèses retrouvent un trouble psychotique ou un trouble de personnalité chez les mères et/ou les pères d'enfants souffrant d'une perturbation de leur sommeil [32, 82, 83].

\section{Description clinique}

\section{Clinique}

Les troubles du sommeil du bébé et du jeune enfant font l'objet d'une classification spécifique éditée par l'Académie Américaine de la Médecine du Sommeil [3]. Ils apparaissent dans les classifications internationales des troubles mentaux, DSM [4, 5] et CIM-10 [103], ainsi que dans la classification nationale des troubles mentaux de l'enfant et de l'adolescent (CFTMEA [97]). Ils représentent une entité bien distincte dans la Classification Diagnostique de la Santé mentale et des Troubles du Développement de la Première et Petite Enfance (CD: 0-3) [136]. Dans la nouvelle version de la classification (CD: 0-3R) publiée en 2005 [137], la catégorie des «Troubles du Comportement du Sommeil», comporte une spécification de ses critères diagnostiques. Sa description intègre des souscatégories et des critères issus du groupe de travail RDC-PA (Research Diagnostic Criteria: Infancy and Preschool, Académie Américaine dePsychiatrie de l'Enfant et de l'adolescent, 2003). Des critères d'âge de début des troubles, d'intensité et de durée apparaissent. En suivant les recommandations de la RDC-PA, deux formes de troubles sont distinguées: les «Troubles de l'endormissement» (code 510) et les «Troubles de la 
durée du sommeil» (code 520) qui complètent les nouvelles catégories du DSM-IV-TR [5]: terreurs nocturnes, somnambulisme, troubles du sommeil liés à la respiration et cauchemars. Si les critères d'une des catégories du DSM-IV-TR sont remplis chez le jeune enfant, alors le diagnostic approprié doit être reporté dans l'Axe I de la CD: 0-3R (code 800). Les critères diagnostiques principaux sont les suivants:

\section{Troubles de l'endormissement}

- Les troubles de l'endormissement se reflètent dans le temps que l'enfant met pour parvenir à s'endormir, son besoin que ses parents demeurent dans sa chambre jusqu'à qu'il soit endormi, et/ou le besoin de l'enfant de rappeler ses parents après qu'ils aient quitté la chambre.

- Le diagnostic de Trouble de l'endormissement implique la présence de difficultés sérieuses d'endormissement pendant au moins quatre semaines, avec cinq à sept épisodes par semaine.

- L'enfant doit avoir 12 mois ou plus.

\section{Troubles de la durée du sommeil}

- Les troubles de la durée du sommeil se manifestent par des réveils nocturnes qui requièrent l'intervention parentale et/ou le déplacement de l'enfant dans le lit des parents.

- Un diagnostic de Trouble de la durée du sommeil implique que des difficultés sérieuses de réveils nocturnes soient présentes pendant au moins quatre semaines, avec cinq à sept épisodes par semaine.

- L'enfant doit avoir 12 mois ou plus.

Enfin, la CD: 0-3R précise que le diagnostic de «Trouble du Comportement du Sommeil» ne devrait pas être utilisé lorsque les difficultés de sommeil sont avant tout dues à un «Trouble des Affects», un «Trouble de l'Ajustement», un «Etat de Stress post-traumatique» ou un «Trouble de la Relation».

\section{Formes cliniques}

A la phase précoce du développement, les insomnies sont les plus fréquentes. L. Kreisler [76, 77] propose une nosographie des insomnies prenant en compte le stade de développement traversé et l'intensité du trouble. 
- Les insomnies communes du premier semestre sont décrites comme d'une grande banalité dans les premiers mois de vie au cours desquels le sommeil subit des modifications majeures, dans ses caractéristiques à la fois biologique et psychologique. Elles sont marquées par des périodes de sommeil brèves, des réveils, des pleurs et des cris fréquents. Il s'agit le plus souvent de conditions inopportunes ou maladroites mais elles peuvent être le témoin de difficultés précoces d'adéquation entre le bébé et sa mère [87]. Il importe de les aborder convenablement car elles peuvent s'installer ou conditionner, peut-être pour longtemps, un sommeil de mauvaise qualité [76].

- Les insomnies précoces sévères [76, 77] se signalent par leur durée, leur intensité, leurs signes d'accompagnement, notamment d'ordre moteur: cris, agitation ou au contraire apathie. Rares dans la pratique clinique quotidienne, L. Kreisler en décrit trois formes principales, avec en commun l'intensité quantitative de l'insomnie (ces bébés ne dorment que 2 à 4 heures sur 24): la grande insomnie tapageuse, l'enfant n'étant qu'un cri jour et nuit; l'insomnie avec activité en décharge motrice de gestes souvent auto-agressifs, étrangement rythmés, intenses et élaborés, compte tenu de l'âge; la grande insomnie silencieuse, ces bébés restant de longues heures du jour et de la nuit, les yeux grands ouverts, silencieux, signe d'un dysfonctionnement interactif grave. Ces insomnies graves et précoces sont fréquemment rencontrées dans l'histoire précoce d'enfants souffrant de pathologie autistique ou psychotique; ainsi, elles comptent au titre des signes d'alarme précoces de ces pathologies.

- Les insomnies de la deuxième et de la troisième année sont rencontrées dans le processus du développement normal de l'enfant. A cet âge, l'enfant est un «excité», avide de connaître, sans cesse en mouvement; l'enfant excité est remuant, instable dans ses intérêts et ses jeux, d'humeur labile. L'angoisse de séparation est inhérente à cette phase du développement. L'endormissement est souvent difficile, marqué par les incidents, le recours aux activités auto-érotiques et aux objets transitionnels, aux rituels d'endormissements. L'enfant est également « inquiet», en aller et retour constant entre l'opposition et la dépendance. Les réveils de l'anxieux expriment la peur, la frayeur du cauchemar, la terreur, l'angoisse de séparation. Il existe des troubles du sommeil, par excitation psychique, qui font parler « d'insomnies joyeuses» qui s'observent soit dans un climat de dépression maternelle, soit chez des enfants psychotiques. 
Les hypersomnies [89] sont très peu fréquentes dans la clinique du jeune enfant. La somnolence diurne excessive, lorsqu'elle est importante, est inquiétante: la somnolence prolongée, le peu de réactivité, l'indifférence, le silence et le calme amènent à envisager une déficience mentale ou un état de retrait autistique précoce. Par ailleurs, à partir de 8-9 mois, certains enfants manifestent au cours de la journée des périodes d'endormissement brutal ou rapide, soit secondaire à une frustration, soit sans raison apparente; elles surviennent volontiers dans un contexte de tristesse et de dépression. Les deux principales parasomnies $[2,18,87,89]$ sont les terreurs nocturnes et les cauchemars. Enfin, les rythmies du sommeil $[18,87,89,77]$ appartiennent aux troubles moteurs liés au sommeil et surviennent le plus souvent dans le processus normal du développement de l'enfant.

L'analyse sémiologique minutieuse du trouble est indispensable. Il est essentiel de différencier un trouble passager, contemporain d'une «mini-crise développementale» [22], considéré comme une variation de la normale, d'un trouble durable. Ainsi, le trouble du sommeil du bébé et du jeune enfant ne prend sa valeur psychopathologique que s'il apparaît en dehors des crises développementales normales et/ou s'il s'installe dans le temps. De même, la qualité du symptôme offre une multitude de nuances qui correspondent chacune à un risque psychopathologique différent.

\section{Caractéristiques tempéramentales et comportementales - Symptômes associés}

De nombreuses caractéristiques tempéramentales ont été associées aux nourrissons et jeunes enfants présentant des troubles du sommeil [9,24, $120,122,129,130]$. En effet, l'organisation des rythmes veille/sommeil semble être déterminée, à un certain degré, par différentes dimensions du tempérament de l'enfant [73]. La majorité des études s'accordent sur l'association entre un tempérament «difficile» et les troubles du sommeil du jeune enfant. Plusieurs traits tempéramentaux sont associés aux durées de sommeil diurne et nocturne: humeur générale de l'enfant, obstination [130], seuil de sensorialité [24], capacité d'approche [122], repli, adaptabilité et distractibilité [120]. Selon T.F. Anders, et al. [9, 54], le tempérament de 1'enfant n'est pas directement lié à la quantité des réveils nocturnes, mais à la propension de l'enfant à les signaler. Les «self-soothers» dorment mieux, et sont décrits comme plus «faciles». Les «signalers» dorment moins, pleurent pour signaler leurs réveils, et sont décrits comme plus «difficiles». 
Ces études confirment la participation de facteurs constitutionnels dans la survenue des troubles du sommeil du bébé et du jeune enfant.

Les difficultés de sommeil du jeune enfant font souvent partie d'une perturbation comportementale plus globale. Certains auteurs [59] retrouvent dans le cas d'insomnies précoces, sévères et prolongées, des bébés avec un comportement d'excitation, voire d'agitation, associé souvent à une avance du développement psychomoteur. L. Kreisler [77] souligne la présence d'une angoisse et d'un état d'excitation. Plus récemment, A. Le Nestour, et al. [83] retrouvent, dans leur cohorte de bébés gravement insomniaques et hyper-agités, des manifestations bruyantes pendant les périodes de veille (cris stridents, longs pleurs), des troubles du tonus (le plus souvent sous la forme hypertonique, parfois jusqu'à l'opisthotonos alternant avec une hypotonie majeure), des mouvements incessants globaux ou, plus souvent, localisés aux membres inférieurs (appui très précoce sur leurs jambes), une avidité orale, une avidité visuelle à tout ce qui bouge.

A. Guedeney et L. Kreisler [59] soulignent la coïncidence en proportion significative avec d'autres désordres psychosomatiques; les plus fréquemment associés étant: coliques du premier trimestre, colon irritable, vomissements, spasmes du sanglot, dermite atopique, troubles respiratoires. Plusieurs études s'accordent sur l'association entre les troubles du sommeil et les troubles de l'alimentation, dans la première enfance [32]. Les troubles du sommeil du bébé et du jeune enfant peuvent s'intégrer dans un tableau plus sévère. C'est le cas des insomnies graves, observées chez des enfants dont le développement présente des zones de fragilité, susceptibles de les faire s'engager dans un fonctionnement ou une organisation de type autistique ou psychotique. Enfin, ces troubles peuvent s'intégrer dans un tableau de dépression du bébé [61].

\section{Facteurs environnementaux}

Les conditions environnementales constituent une orientation étiologique essentielle. Même si elles sont rarement isolées, leur exploration doit être systématique $[77,89]$. Elles concernent beaucoup d'insomnies communes du premier semestre, souvent liées à une puériculture erronée: les erreurs diététiques sont les plus fréquentes [77], la méconnaissance des rythmes de l'enfant et la rigidité des horaires du coucher participent également à la survenue des troubles. Beaucoup de travaux se sont orientés sur les interventions parentales au moment du coucher et en réponse aux réveils nocturnes de l'enfant $[8,73,129,138]$. La présence continue des parents 
au moment du coucher est considérée comme une «protodyssomnie» dans une nouvelle et récente classification des troubles du sommeil de l'enfant $[27,80]$. Toutes les techniques de réconfort qui se font en dehors du lit de l'enfant (alimentation, bercement) sont associées à une consolidation plus fragile du sommeil. Elles s'interposent comme substitut aux capacités inhérentes du bébé, dont celle d'autoapaisement, appelée «selfsoothing» par T.F. Anders [9,54]; c'est cette capacité qui permet au bébé de trouver la tranquillité nécessaire à son endormissement, mais également de se rendormir seul lors d'éveils nocturnes spontanés. Enfin, l'agitation, le bruit, le surpeuplement du logement peuvent être à l'origine de troubles du sommeil [77].

\section{Diagnostics différentiels}

La recherche d'une perturbation organique fait partie de la démarche étiologique nécessaire à la prise en charge des troubles, à cette phase précoce du développement. Certains symptômes et données de l'examen clinique doivent faire suspecter une étiologie organique [18, 26, 27]: éveils longs, temps de sommeil très diminué, éveils de première partie de nuit, hyperactivité diurne anormale, troubles alimentaires, régurgitations anormales, ronflement anormal, hypersudation, retard staturo-pondéral, ou cassure de la courbe de poids et enfin examen neurologique ou psychomoteur anormal.

Dans la petite enfance, on pensera essentiellement à l'épilepsie avec crises nocturnes [80]. Quatre maladies génétiques sont presque systématiquement associées à des insomnies graves liées à des troubles spécifiques de l'installation du rythme circadien et/ou des anomalies de la structure du sommeil et des apnées du sommeil [36, 37]: le syndrome de Smith-Magenis, le syndrome d'Angelman, le syndrome de Prader-Willi, et le syndrome de Rett. Les troubles digestifs sont dominés par le reflux gastro-œsophagien qui favorise les éveils nocturnes [27, 80]. Le syndrome d'apnée obstructive du sommeil [18] représente, avec les étiologies neurologiques, une des causes les plus fréquentes d'excès de sommeil diurne du jeune enfant. Toute pathologie infectieuse ou algique $[27,80]$ peut être à l'origine d'une perturbation du sommeil du jeune enfant; enfin, plus rares, les causes toxiques, médicamenteuses [71].

\section{Principaux axes étiopathogéniques}

De nombreuses hypothèses ont été avancées dans la littérature quant à l'origine et à la signification des troubles du sommeil du bébé et du jeune 
enfant. Il nous paraît essentiel de préciser d'emblée, qu'à cette phase précoce du développement, ces troubles ne doivent pas être considérés comme un symptôme propre au bébé mais comme un symptôme interactif, ou encore le symptôme d'une constellation familiale. Après une analyse sémiologique précise de ce symptôme, l'évaluation psychopathologique nécessite une exploration fine des interactions entre le bébé et ses parents. Les perturbations d'ordre relationnel et conflictuel que les troubles du sommeil reflètent sont d'une extrême diversité. Notamment les interactions peuvent se situer dans des registres variés en fonction de la structure de personnalité parentale, voire survenir dans le cadre d'une pathologie psychiatriqueassociée.

\section{Les troubles du sommeil: symptôme psychosomatique}

Chez le bébé, qui ne dispose pas encore du langage, le corps est le lieu d'expression privilégié de la souffrance psychique. Dans la clinique psychosomatique du bébé et du jeune enfant, le trouble du sommeil occupe une "position centrale en raison de sa fréquence, de ses incidences, et en tant que modèle de compréhension du trouble psychosomatique précoce» [76, 77].

Les thèses des psychanalystes psychosomaticiens mettent l'accent sur la relation entre la fonction maternelle et le sommeil de l'enfant. Selon les travaux fondateurs de L. Kreisler, M. Fain, M. Soulé [76] sur la pathologie psychosomatique du bébé, le sommeil et sa pathologie constituent une illustration de la théorie de l'étayage au sens de S. Freud [48], autour de l'infiltration pulsionnelle de la fonction hypnique. A ce stade archaïque, la fonction de «gardien du sommeil», plus tard assurée par le rêve, est étayée par la mère. Dans les cas où la mère n'accomplit pas sa fonction de libidinalisation secondaire, le sommeil peut garder les caractéristiques des premiers temps lorsqu'il est provoqué physiologiquement par la satiété qui suit l'allaitement. Ainsi, le sommeil manque à son rôle de protection quand la mère manque à sa fonction d'étayage de la fonction hypnique. Toujours en s'appuyant sur les travaux de S. Freud [49], ces auteurs postulent qu'il existe chez le nourrisson gravement insomniaque un blocage du processus hallucinatoire primaire, première élaboration mentale, dont l'activité onirique est l'héritière directe.

\section{Problématique de la séparation}

L'hypothèse qui est largement développée dans la littérature, concerne bien sûr la problématique de l'élaboration de la séparation dans la relation parent-enfant. Selon B. Cramer[30,31], «les difficultés d'endormissement 
et les réveils nocturnes peuvent s'expliquer par des difficultés à négocier la séparation pour l'enfant et ses parents».

Du côté du bébé, l'angoisse de séparation fait partie du développement affectif normal. Du fait de sa réémergence au moment du coucher, elle occupe une place centrale dans la compréhension des troubles. En effet, la séparation imposée par le coucher et l'extinction de toutes les sensations diurnes vont être angoissantes pour le bébé qui les vit comme irréversibles: "Dès l'instant qu'il perd de vue sa mère, il se comporte comme s'il ne devaitjamais plus la revoir» (S. Freud). L'investissement maternel joue un rôle majeur dans la gestion de cette angoisse, notamment à travers la mise en place des capacités d'autoapaisement du bébé. Pour certains auteurs [76, 127], certains modes de réponses aux besoins de l'enfant s'opposent à l'établissement des processus autoérotiques et hallucinatoires. Les réveils nocturnes de l'enfant viennent traduire son incapacité à halluciner dans un rêve la satisfaction que le sein peut donner. La mère reste «le gardien du sommeil» et ne cède pas sa place au rêve [76]. Les situations sont en fait très variées. C'est le cas de certains allaitements prolongés dans le temps, où l'érotisme ne peut passer que par le sein maternel, le bébé ne pouvant développer le sien propre. Au maximum, l'enfant «fœetalisé», repu et sans désir, relié au sein maternel comme à un cordon ombilical, reste dans un mode de vie évocateur du narcissisme originaire dans lequel il est incapable de développer ses autoérotismes [127]. Dans d'autres cas, il peut s'agir d'une lutte anti-plaisir, la mère n'acceptant pas de différencier le bébé comme un sujet avec son propre plaisir et empêchant son autosensualité. Ailleurs une mère déprimée peut utiliser son bébé pour combler son vide interne; l'enfant devient le remède de sa dépression. Selon B. Golse [53], bien souvent, les manœuvres d'autoapaisement et de réendormissement du jeune enfant sont empêchées par l'angoisse parentale.

Chez le bébé insomniaque un peu plus grand, on fait référence à une insuffisance, voire à l'absence, de l'«espace et de l'objet transitionnel» [40]. Certaines mères, en étant dans un «collage» continu avec leur bébé, ne permettent pas l'établissement de cette aire transitionnelle. Dans d'autres situations [52], si la mère ou son représentant s'absente pour une période qui dépasse la propre capacité de l'enfant de maintenir vivante sa représentation par le recours à l'objet transitionnel, celui-ci ne pourra plus jouer sa fonction symbolique de trait d'union. Il perdra petit à petit sa signification et sera désinvesti. A l'extrême, l'«objet transitionnel» sert de déni de la séparation d'avec la mère. C'est le cas d'enfants qui vivent dans 
une grande insécurité, tourmentés par l'idée de la séparation et de la perte, et qui cherchent à la nier au moyen du lien avec un objet transitionnel pathologique.

Le rapprochement du couple parental, que le sommeil de l'enfant va permettre, le confronte à sa " capacité d'être seul» [131], celle-ci prenant sa source à partir de «l'expérience d'être seul en tant que nourrisson et petit enfant, en présence de la mère». La sécurité que la mère offre à son enfant rend possible une expérience positive de solitude pour un temps limité. L'enfant intériorise la mère, support du Moi, ce qui lui permet d'être effectivement seul: «le bon objet total est intériorisé». Un sommeil paisible nécessite l'établissement d'une continuité d'existence des objets internes du bébé, mais aussi des représentations de lui-même que portent ses parents [15].

S'endormir c'est aussi offrir un manque de soi à l'autre. Certains bébés développent des troubles du sommeil devant cette impossibilité pour leur entourage à tolérer ce manque d'eux [15]. Ainsi, dans la problématique des troubles du sommeil du jeune enfant, les difficultés de séparation psychique des parents sont souvent au premier plan. Les angoisses mortifères des parents, liées à des deuils périnataux encore très douloureux, et/ou face au risque de mort subite, rendent toute absence assimilable à une perte $[11,31,40]$. Ailleurs, la difficulté pour les parents à gérer les situations de désinvestissement peut s'originer dans une problématique de séparation et d'abandon dans leur propre enfance [40]. Ces traumatismes du passé sont réactivés par la naissance de l'enfant. Toute séparation avec ce dernier est alors insupportable car ses pleurs sont interprétés comme l'expression d'une souffrance, et les parents ont peur d'être «traumatisants» en laissant pleurer leur bébé. Ou encore, ces angoisses peuvent être réactualisées et/ou intensifiées par toutes les situations de séparation auxquelles l'enfant et ses parents peuvent être confrontés dans la réalité. Il en est ainsi, de la nécessité impérative de se séparer le jour. Laisser (abandonner) son bébé pour aller travailler peut susciter chez certaines mères des attitudes de compensation; du fait de leur culpabilité, elles acceptent « un travail de nuit» auprès de leurs enfants [11]. Une conjoncture souvent mentionnée est celle où l'enfant se sent trop massivement désinvesti par une mère trop endeuillée [32, 40, 61]. Un deuil non élaboré contemporain d'une grossesse, de la naissance d'un enfant, ou plus ancien dans l'histoire infantile des parents, peut entraver l'investissement maternel et avoir des répercussions sur le sommeil. A. Green [57] désigne ces enfants sous le terme d' «enfants de la mère morte», chez lesquels, la mère, en 
deuil au moment de la naissance, est indisponible pour eux. L'enfant va venir représenter, pour le parent, cet être cher disparu, et donc, permettre à la mère d'éviter un véritable travail de deuil qui risquerait d'avoir pour elle la signification d'un oubli, ou d'une trahison [2]. Lorsque les mères sont déprimées en période post-natale, le bébé développe aussi très fréquemment des troubles du sommeil $[59,60]$. La rêverie maternelle, ici, a laissé la place à un vide de pensée, à un blanc de représentation que seule la réalité du bébé peut combler. Il n'est pas possible à celui-ci alors de laisser sa mère disparaître par sa propre absence [15].

Pour d'autres auteurs, ce qui est déterminant, c'est la façon dont la mère peut elle-même gérer les désinvestissements et permettre à son enfant de la désinvestir dans l'état de veille ou de sommeil, et favoriser chez lui des représentations psychiques lui permettant de supporter son absence. M. Fain [21] trouve dans le bercement du nourrisson par sa mère, au moment de l'endormissement, l'exemple paradigmatique de sa conception psychanalytique de la «censure de l'amante». Le renoncement du bébé à la relation vigile avec sa mère dépend d'abord de la façon dont elle l'a préparé à travers le message qu'elle lui transmet lors de l'endormissement, par exemple dans le bercement. Selon lui, le message maternel est double: il transmet au bébé la nécessité de dormir pour conserver sa santé et avoir un développement satisfaisant, mais aussi, en dormant, de permettre à la mère d'avoir d'autres investissements que lui, c'est-àdire renouveler la scène primitive avec son partenaire sexuel. Finalement, que le désinvestissement au moment de la séparation du coucher soit vécu comme trop massif ou au contraire comme insuffisant, le tableau clinique d'insomnie est comparable dans les deux cas. Il est marqué par le retour d'un état de tension qui ne se décharge pas et ne s'apaise pas [127]. Ainsi, selon L. Kreisler, la grande insomnie d'excitation du bébé peut avoir des caractéristiques qui la rapprochent d'un état provoqué par la répétition d'un trauma. L'expression de «mère intricante», utilisée par D. Ribas [127], résume les variations que subit l'investissement d'un enfant par une mère qui n'en reste pas moins femme: «Lafonction d'intrication pulsionnelle est déterminée par l'intrigue amoureuse dont cet enfant est issu et évincé en même temps». Lorsque la mère n'est pas suffisamment intricante, l'endormissement peut devenir une situation traumatogène où prédomine la désintrication pulsionnelle. L'insomnie précoce peut, en effet, être interprétée, du moins dans certains de ses aspects, comme la conséquence de la désintrication ou de la non-intrication pulsionnelle de l'étayage [76, 127]. Ainsi, trop d'investissement semble équivalent au désinvestissement. L'excès 
de liaison des pulsions peut avoir un impact aussi traumatique qu'une insuffisance de liaison.

Enfin, pour beaucoup d'auteurs, les troubles du sommeil sont une illustration exemplaire de la pathologie psychosomatique liée au surplus d'excitation, lui-même lié à la faillite de la fonction maternelle de pareexcitation $[76,77,59,60,89]$. Les caractéristiques sensorielles et mentales du nouveau-né lui assurent une certaine protection contre les excitations extérieures. L'« habituation» permet au bébé de moduler la quantité d'excitation que son psychisme doit traiter, évitant ainsi le débordement par un afflux massif de sollicitations internes et externes. Mais s'il possède une telle fonction, elle reste, dans les débuts, immature et inopérante $[2,60,76]$. La mère doit être capable de créer un environnement protecteur autour de l'enfant. Ainsi, les mères d'enfant présentant des troubles du sommeil peuvent ne pas accomplir leur rôle de pare-excitation ou le faire de façon inadaptée. B. Cramer [31] précise que c'est leur propre excitabilité interne qui les empêche d'être réceptives à l'enfant, et principalement à son besoin d'être calmé et entouré au moment de la régression imposée par le sommeil. Selon P. Mazet et S. Stoleru [89], un surplus d'excitation peut provenir d'une mère trop soucieuse du bon développement de son enfant. Celle-ci le soumet à de multiples sollicitations afin de conforter son image du bébé idéal. Pour B. Golse [53], la faillite de la fonction maternelle de pare-excitation est liée à une insuffisance de la « capacité de rêverie» de la mère, expression empruntée à la théorie bionienne [19]. A. Le Nestour, et al. [82, 83] posent l'hypothèse du défaut de contenance d'excitation physique des bébés gravement insomniaques et excités, lié à des défaillances d'enveloppes psychiques parentales. Enfin, selon G. Szwec [127], un excès de pare-excitation maternel peut entraver chez un bébé, la constitution d'un pare-excitation interne chargé d'en prendre le relais. L'indisponibilité, les évitements, les incompréhensions du côté maternel favorisent la discontinuité dans la relation avec l'enfant, et sont à l'origine de dysfonctionnements. La mère alterne des périodes de stimulation excessive de l'enfant ou de rapprochement fusionnel, et des périodes de rejet [77]. Ainsi, la surcharge d'excitation et les distorsions représentent la pathologie psychosomatique du surplus d'excitation, du défaut de pare-excitation et du conflit.

Ces comportements complexes, parfois pathogènes, renvoient aux problématiques complexes de la mère et plus globalement aux problématiques parentales qui se rejouent dans l'interaction avec l'enfant. Les mécanismes de régulation du bébé, les phénomènes d'habituation et de 
filtrage qui font partie de son système de pare-excitation, entrent en résonance avec la vie fantasmatique des parents.

\section{La transmission transgénérationnelle}

Les processus psychologiques qui ont lieu normalement chez les mères (et les pères) lors de la grossesse, la naissance de l'enfant et l'émergence de la parentalité, vont constituer l'arrière-fond sur lequel des troubles du sommeil du bébé peuvent apparaître. La période périnatale est une phase pleine de vicissitudes et une terre fertile pour les projections inconscientes des parents. Ceux-ci sont confrontés à des remaniements identificatoires profonds, dictés par la reviviscence de conflits anciens. Pour certains auteurs, certaines projections parentales peuvent entraver les processus d'harmonisation des premiers liens entre la mère et son bébé. Celui-ci peut devenir le réservoir des projections des conflits inconscients de ses parents. Les troubles du sommeil du bébé et de l'enfant se comprennent alors à la lumière des problématiques anaclitiques et œdipiennes des parents. C'est en s'inspirant des travaux de B. Cramer [29] sur la psychothérapie brève que F. Palacio-Espasa, J. Manzano et N. Zilkha [86] ont approfondi la notion de projections parentales sur 1'enfant. La projection des parents sur l'enfant constitue plus exactement une identification projective; elle fait partie du travail d'instauration de la parentalité, et de la construction du lien parent-enfant. Ensemble et séparément, les parents attribuent au bébé des caractéristiques ayant un sens et un lien avec leur propre histoire et leur passé. Le bébé fantasmatique, fruit des désirs œdipiens, réveille une conflictualisation et constitue un travail de parentalité d'emblée conflictuel et ambivalent. En effet, bien que les parents externalisent en projetant de manière identificatoire leur propre narcissisme infantile sur leur bébé, ils projettent aussi, à leur insu, des parties d'eux-mêmes, enfant, plus négatives ou conflictuelles, ou des parties d'imagos parentales, elles-mêmes conflictuelles. C'est ce que J. Manzano et F. PalacioEspasa ont appelé: «L'ombre de soi des parents projetée sur l'enfant ou l'ombre de l'objet des parents projetée sur l'enfant». Les auteurs ont analysé les projections parentales qui pèsent sur l'enfant selon les différentes configurations des ombres qui pèsent sur lui. Lorsque les reproches que le parent peut faire à son propre parent sont liés à une problématique d'abandon ou de séparation, le parent va avoir tendance à vouloir « réparer» tout mouvement de séparation du bébé avec lui. Tous les pleurs et les cris du bébé seront interprétés comme un appel, une souffrance, la nuit surtout. Cette problématique d'abandon, va faire collusion avec la pro- 
blématique œdipienne de «laisser l'enfant pour que les parents se retrouvent ensemble». Il y a donc réactivation des deux niveaux de conflictualités névrotiques. Finalement les appels de l'enfant la nuit ont pour rôle essentiel de mobiliser autour du berceau tant les parents que les fantômes du passé, les fantômes de la chambre d'enfant [43]. Les troubles du sommeil du bébé deviennent alors le symptôme de la problématique familiale inconsciente.

Les processus d'attachement ont bien sûr été reliés aux problèmes de sommeil des jeunes enfants. Des travaux mettent en évidence l'existence d'une transmission transgénérationnelle des modalités d'attachement $[1,62,85]$ : les expériences émotionnelles vécues dans l'enfance sont à l'origine d'un ensemble de représentations internes, les «modèles internes opérants», qui déterminent chez l'enfant son type d'attachement; les «modèles internes opérants» déterminent chez l'enfant, devenu parent, un ensemble de modalités comportementales qui vont à leur tour déterminer les représentations internes du nouvel enfant. Selon le « modèle d'attachement inversé» [98], si les parents sont eux-mêmes anxieux par rapport à leurs propres figures d'attachement, ils inversent la relation parent-enfant, exigeant de l'enfant qu'il représente l'image parentale. C'est à l'enfant qu'incombe la responsabilité de rester disponible pour la mère. Dans une relation d'attachement normale, les mères de jeunes enfants restent toujours «de garde» pendant la nuit. Dans le cadre d'un attachement inversé, le jeune enfant ne parvient pas à dormir profondément car il a le sentiment d'être responsable de sa mère. Ce sentiment d'insécurité, dont l'enfant ne peut se défaire, l'oblige à rester vigilant.

B. Cramer [31] propose que la transmission transgénérationnelle, vue sous le prisme de la théorie de l'attachement, puisse rendre compte de la réémergence des troubles à d'autres phases du développement. La permanence d'un modèle interne d'insécurité relationnelle, inscrit dans la psyché comme un programme déterminant les réactions relationnelles, assure la continuité de la susceptibilité à l'angoisse, codéterminant ainsi l'apparition des symptômes.

\section{Etiologie culturelle}

Si le sommeil est universellement synonyme de séparation et de mort, chaque culture accompagne, selon ses modalités propres, le jeune enfant lors de son endormissement. Deux styles d'interaction mère-enfant sont décrits dans la pratique de l'endormissement [125]. L'interaction distale privilégiée dans les sociétés occidentales, implique la prédominance du 
regard et de la voix au détriment du toucher et favorise l'isolement de l'enfant. A l'opposé, l'interaction proximale, impliquant la prédominance du toucher, est privilégiée dans les sociétés traditionnelles extra-occidentales; dans ces cultures, mère et bébé dorment ensemble. Ainsi, si une corrélation entre le trouble du sommeil et le mode de coucher a été établie, elle fait encore l'objet d'un débat qui oppose les chercheurs épidémiologistes occidentaux et les chercheurs cliniciens transculturels. Pour les premiers $[27,55,56,125]$, le contact de proximité mère-enfant lors du coucher, est pourvoyeur de trouble du sommeil. Pour les seconds [55, 56, 125], à l'inverse, le contact de proximité ne favorise pas les troubles du sommeil; bien au contraire, la vigilance qui entoure le sommeil de l'enfant contribue à éviter ce problème. Pour Y. Govindama [55, 56], ce débat ne prend pas en compte les croyances culturelles sous-jacentes à ces pratiques du coucher. Ce n'est pas la pratique du coucher qui est en cause, mais la représentation culturelle du sommeil et de son trouble, propre à chaque société. C'est elle qui détermine le mode d'accompagnement de l'enfant dans la séparation d'avec sa mère. Les croyances culturelles viennent faire tiers entre la mère et son bébé. Dans les sociétés extra-occidentales, la pratique du co-sleeping, comme mode de coucher, repose non seulement sur la prise en compte de l'immaturité psychobiologique du bébé, mais surtout sur l'idée que son âme est encore trop fragile pour le protéger des attaques des mauvais esprits. Le co-sleeping détient un sens culturel. Il sert à protéger l'enfant de la solitude, de son immaturité "psychobiologique» l'exposant à «la mort réelle ou à la mort psychique (la folie), car il peut être possédé par un être invisible, possession qui se manifeste à travers les troubles du sommeil». Y. Govindama montre que le co-sleeping s'inscrit dans une perspective de prévention du trouble lorsqu'il est médiatisé par la culture, mais que le geste lui-même, lorsqu'il est dépourvu de sens, est pourvoyeur de trouble indépendamment des cultures. Dans un contexte migratoire, la pratique du co-sleeping peut engendrer des troubles si elle a perdu son sens culturel premier. Cette technique peut être utilisée par certaines mères migrantes, non pas pour protéger l'enfant du monde de la nuit, mais pour combler un vide interne, s'autoapaiser face à une anxiété débordante et se défendre contre un possible effondrement dépressif. Ce mode de contact favorise, dans ce cas, non seulement un trouble du sommeil, mais développe une dépendance pathologique chez l'enfant. On assiste alors à un déni de la différence des générations et des sexes compromettant pour l'altérité de l'enfant. 


\section{Prise en charge et traitement}

La prise en charge des troubles du sommeil de l'enfant est diversifiée. Le type de thérapeutique proposée dépend essentiellement de l'orientation du thérapeute. Le traitement peut associer, selon les cas et selon les auteurs, une psychothérapie psychodynamique mère-bébé ou parent-bébé, une thérapie familiale, une psychothérapie comportementale avec guidance interactive, et, dans des cas plus exceptionnels, un traitement médicamenteux. Certains auteurs préconisent l'utilisation d'un agenda de sommeil [27, 81] permettant de confirmer les données de l'anamnèse, celle-ci constituant une étape fondamentale [27, 31, 32, 77] de la prise en charge. A partir de cet agenda, utilisé comme référence tout au long du traitement, un certain nombre de mesures d'hygiène de sommeil sont proposées. Même si l'amendement des troubles du sommeil est le but premier de la prise en charge, il ne constitue pas une fin en soi. Il nous paraît indispensable de s'intéresser au sens du symptôme dans l'interaction mère-bébé (mère-père-bébé) ainsi que dans l'histoire familiale.

Les troubles psychofonctionnels du bébé et du jeune enfant sont une indication majeure des psychothérapies conjointes mère-bébé ou parentbébé [29, 30, 31, 74, 75]. L'hypothèse présupposée est que les troubles du sommeil du nourrisson trouvent leur origine, d'une part, dans l'impact exercé par les projections et les représentations maternelles ou parentales sur le fonctionnement du bébé et, d'autre part, dans les distorsions relationnelles précoces. Les psychothérapies conjointes parent-bébé ont une action thérapeutique mais aussi préventive. En effet, elles cherchent non seulement à produire des effets sur les symptômes amenés lors de la consultation, mais elles ont aussi une action préventive sur la constitution possible d'une conflictualité intrapsychique figée de l'enfant.

Dans le travail avec les bébés, selon la méthode psychodynamique, deux grands axes de soins sont proposés: les psychothérapies brèves, mère-bébé ou parent-bébé, et les traitements conjoints au long cours. Les objectifs du traitement psychanalytique consistent à déterminer, d'une part, comment l'enfant s'inscrit dans l'histoire transgénérationnelle des parents, et d'autre part, par quels mécanismes la conflictualité intrapsychique parentale se matérialise dans la relation à l'enfant, par le biais d'interactions comportementales qui entraînent une manifestation symptomatique chez le bébé, avant même que ne se soit fixée une problématique intrapsychique chezlui.

La guidance interactive $[2,74]$ se focalise sur l'interaction visible et en cours, dans une attitude thérapeutique de base positive et encourageante, 


\section{Résumé}

Les troubles du sommeil de l'enfant constituent un des principaux motifs de consultation de pédiatrie et pédopsychiatrie. Par leur fréquence et leur retentissement sur la vie familiale, ils se trouvent au centre de la clinique fonctionnelle de la petite enfance. Les caractéristiques du sommeil évoluent très rapidement lors des premiers mois de la vie. La connaissance de cette ontogenèse du sommeil est indispensable pour identifier les perturbations « authentiques» du sommeil, et les différencier des caractéristiques normales d'un sommeil en pleine maturation. Le sommeil du bébé ne se résume pas à un développement neurophysiologique. II est en étroite relation avec les soins maternels qui l'imprègnent de sa charge affective. La pathologie du sommeil s'organise dans la sphère des interactions précoces entre le bébé, la mère et le père. Après une analyse sémiologique précise du symptôme, l'évaluation psychopathologique nécessite une exploration fine des interactions entre le bébé et les parents. Depuis plusieurs années, certains auteurs où l'usage de la vidéo permet à la mère ou aux parents de visualiser leurs comportements interactifs avec le bébé. Dans cette approche, il n'y a pas de référence explicite à l'histoire de la mère ou des parents, et le thérapeute commente les séquences interactionnelles vécues dans «l'ici et le maintenant», dans le but de renforcer, par ses encouragements, les interactions qui se révèlent favorables à une harmonisation de l'interrelation. L'efficacité de cette forme d'intervention a été prouvée. Les études font la preuve d'une utilité des processus thérapeutiques, autant suivant la méthode psychodynamique que l'approche de guidance interactive.

L'approche comportementale a été particulièrement développée aux Etats-Unis et elle est très pratiquée par les pédiatres [41]. Il s'agit, selon des protocoles standardisés, d'aider les parents à apprendre à leur enfant à s'endormir et se rendormir seul $[44,45,96]$. Certaines interventions comportementales ont été intégrées à des programmes d'éducation préventive proposés aux parents en devenir et aux parents de nouveau-nés. Celle-ci se focalise sur la mise en place précoce d'habitudes de sommeil positives. Les techniques cognitives ont pour objet de modifier les idées et les croyances erronées sur le sommeil des parents. Ces croyances sont à l'origine de comportements inadaptés et sont associées à un sommeil de mauvaise qualité chezl'enfant [119]. L'efficacité des approches cognitivocomportementales est maintenant bien reconnue pour la grande majorité des troubles du sommeil du jeune enfant (difficultés d'endormissement et réveils nocturnes), avec des taux élevés de réussite, des résultats rapides et des changements sûrs et durables $[44,45,96,105,114,119]$.

Les indications de la thérapie familiale [17, 41, 70, 101], selon une perspective théorique systémique ou psychanalytique, sont basées sur l'existence d'une demande familiale, c'est-à-dire d'une souffrance exprimée et ressentie de façon intense par les parents ou au moins l'un d'entre eux, leconstatd'un dysfonctionnement familial majeurou d'une psychopathologie familiale importante. M.P. Durieux et J.P. Matot [41] préconisent la thérapie familiale au long cours dans les situations de dysfonctionnements familiaux graves, pour permettre l'élaboration progressive d'un processus d'individuation et d'autonomisation entravé par la fragilité du narcissisme des parents.

La prescription médicamenteuse ne doit être qu'un geste d'appoint pour un petit nombre de situations bien précises [89]. Elle ne doit jamais être la réponse unique [107]. La majorité des auteurs [27, 81, 107, 110] s'accordent sur une prescription médicamenteuse ponctuelle (n'excédant pas 4 semaines et si possible 8 à 10 jours), à la dose minimale efficace et sous 
contrôle médical strict, en association à des mesures d'hygiène de sommeil et à une psychothérapie. Deux familles sont principalement concernées: les benzodiazépines et les antihistaminiques [81, 106, 110]. Ces substances ont un effet anti-éveil mais ne sont pas dénuées d'effets sur l'architecture du sommeil $[81,110]$. Le sommeil médicamenteux peut être amputé d'une part importante de ses phases paradoxales. La vigilance diurne est affectée. Les risques d'accoutumance et de dépendance sont importants. Par contre, la mélatonine exogène a montré son intérêt dans le traitement des troubles du sommeil des enfants présentant des troubles neurologiques et comportementaux [36, 37]. Des études ont été menées sur son utilisation dans les maladies génétiques: le syndrome de Rett, le syndrome de Smith-Magenis et le syndrome d'Angelman, ainsi que dans les tumeurs et les lésions de l'hypothalamus et du chiasma optique.

\section{Pronostic -Evolution}

Les études longitudinales révèlent la fréquence élevée de la persistance et de la récurrence des troubles du sommeil du jeune enfant pendant les premières années de vie. Plusieurs facteurs y sont associés. Les données concernant la dépression maternelle sont contradictoires. Pour certains auteurs [138], elle est le seul facteur associé à la persistance des troubles dans l'enfance. D'autres auteurs [72] retrouvent la présence d'un stress dans l'environnement de l'enfant; les plus fréquents étant une absence inhabituelle de la mère, des épisodes de dépression maternelle, une maladie ou un accident chez l'enfant ou sa famille. D. Benoît, et al. [16] retrouvent une association entre la persistance des troubles et un modèle d'attachement insécure des mères. Enfin, l'apparition précoce et l'absence de prise en charge sont des facteurs de risque de passage à la chronicité et d'aggravation des troubles.

De nombreuses études s'intéressent aux effets à long terme d'un sommeil précoce interrompu et/ou insuffisant. Le retentissement sur les domaines cognitif, comportemental et émotionnel est exploré. La comorbidité à long terme est dominée par les problèmes comportementaux, et plus spécifiquement les comportements agressifs [79] et les crises de colère [138]. Des résultats préliminaires [128] retrouvent une association entre troubles du sommeil précoces, sévères et durables et trouble déficit de l'attention/hyperactivité. Sur le plan émotionnel, une irritabilité chronique et une faible modulation des affects sont rapportées. Une dette chronique de sommeil participe également à l'apparition de troubles cognitifs, rapportent des perturbations du sommeil d'une sévérité accrue et survenant chez des enfants de plus en plus jeunes. Les hypothèses étiopathogéniques de ce trouble sont multiples. Les perturbations d'ordre relationnel et conflictuel, qu'il reflète, sont d'une extrême diversité, des plus communes aux plus sévères. Le pronostic de ces troubles est dominé par le passage à la chronicité (persistance ou récurrence). Le retentissement à long terme d'un sommeil précoce insuffisant sur les domaines cognitif, comportemental et émotionnel, est de plus en plus exploré. La prise en charge des troubles du sommeil du bébé et du jeune enfant est diversifiée. Selon les cas et selon les auteurs, il peut s'agir d'une psychothérapie psychodynamique mère-bébé ou parent-bébé, d'une psychothérapie comportementale avec guidance interactive, d'une thérapie familiale, associées, dans des cas plus exceptionnels, à un traitement pharmacologique.

\section{Mots-clés}

Troubles du sommeil. Interactions parent-bébé. Hypothèses étiopathogéniques. 


\section{Summary}

Infant's sleep problems represent one of the most frequent complaints of parents consulting a paediatrician and a psychiatrist. Due to their frequency and family life impact, they are an essential part of infancy functional clinical evaluation. Sleep characteristics develop very quickly over the first few months. The knowledge of their ontogenesis is very important in order to identify «true» sleep disorders from normal maturational sleeping patterns. Infant's sleep does not boil down to neurophysiologic development alone, it is closely linked to the mother's caregiving which implies a strong emotional aspect. Sleep disorders appear as parent-infant interactions organize. After an accurate clinical analysis of the symptom, psychopathological assessment consists of exploring parentinfant interactions. In the last years, some authors have observed a higher severity level of sleep problems in even younger children. Many en particulier des fonctions exécutives [129]. Les fonctions cognitives régulées par le cortex préfrontal, comme la flexibilité cognitive et la capacité à raisonner et penser de façon abstraite, sont particulièrement sensibles aux effets d'un sommeil altéré [96]. Enfin, des difficultés dans les apprentissages sont rapportées chez les enfants d'âge scolaire [129, 134]. De façon plus globale, des effets sur l'état de santé général de l'enfant sont rapportés(fonctions métaboliques et immunitaires, blessures accidentelles) [32, 79, 96].

\section{Conclusion}

La pathologie du sommeil s'organise dans la sphère des interactions précoces entre le bébé, la mère et le père, et doit être considérée non comme un symptôme propre au bébé, mais comme un symptôme interactif. Après une analyse sémiologique précise de ce symptôme, l'évaluation psychopathologique nécessite une exploration fine de ces interactions. Les hypothèses étiologiques de ce trouble sont multiples: symptôme psychosomatique, angoisses de perte et de séparation tant du côté du bébé que des parents, transmission transgénérationnelle, projections parentales, attachement insécure, etc. Les perturbations d'ordre relationnel et conflictuel, que les troubles du sommeil reflètent, sont d'une extrême diversité, des plus communes aux plus sévères. Leur pronostic est dominé par le passage à la chronicité et des données grandissantes montrent qu'un sommeil précoce interrompu et/ou insuffisant a des effets délétères sur le développement de l'enfant, dans les domaines cognitif, émotionnel et comportemental. Sans oublier, bien sûr, la part personnelle que le bébé intrique, dès la naissance, dans la rencontre avec ses partenaires, nous souhaitons traduire, d'une part, comment ce symptôme fonctionnel constitue un modèle de l'effet de la relation sur le système d'autorégulation du bébé et, d'autre part, comment il permet de réinterroger nos modèles psychopathologiques.

[Reçu 10.12.2009, accepté 22.12.2009]

\section{Références}

[1] AINSWORTH M, BREHAR MC, WATERS E, WALL S. : Patterns of attachment. A psychological study of strange situation. Lawrence Erlbaum Ass., Hillsdale, New Jersey, 1978.

[2] ALVAREZ L, GOLSE B.: La psychiatrie du bébé. Que sais-je? PUF, 2008.

[3] AMERICAN ACADEMY OF SLEEP MEDICINE. International Classification of Sleep Disorders, Second Edition (ICSD2). Westchester IL, American Academy of Sleep Medicine, 2005.

[4] AMERICAN PSYCHIATRIC ASSOCIATION. Diagnostic and Statistical Manual of Mental Disorder. $4^{\text {th }}$ edition (DSM IV), Washington DC, American Psychiatric Association, 1994. 
[5] AMERICAN PSYCHIATRIC ASSOCIATION. Diagnostic and Statistical Manual of Mental Disorder. $4^{\text {th }}$ edition revised (DSM IV-TR), Washington DC, American Psychiatric Association, 2001. [6] ANDERS T.F.: «Night-waking in infants during the first year of life », Pediatrics, 1979; 63 (6): 860-864.

[7] ANDERS T.F.: «Neurophysiological studies of sleep in infants and children», Journal of Child Psychology and Psychiatry, 1982; 23 (1): 75-83.

[8] ANDERS T.F, KEENER M.: « Developmental course of night-time sleep wake patterns in full-term and premature infants during the first year of life ", Sleep, 1985; 8: 173-192.

[9] ANDERS T.F, HALPERN L.F, HUA J. : « Sleeping through the night: a developmental perspective», Pediatrics, 1992 ; 9: 554-560.

[10] ANDERS T.F., EIBEN L.A. : "Paediatric sleep disorders: a review of the past 10 Years ", American Academic Journal of Child and Adolescent Psychiatry, 1997, 36 (20) : 9-20.

[11] APTER-DANON G. : "Les bébés dorment, les parents rêvent », Enfances \& Psy, La nuit, $2000 ; 10: 73-76$.

[12] ARCHBOLD K.H. : « Symptoms of sleep disturbances among children at two general paediatric clinics», The Journal of Pediatrics, 2002.

[13] ARMSTRONG K.L., O'DONNEL H., MCCALLUM R., DADDS M. : « Childhood sleep problems: association with prenatal factors and maternal distress/depression», Journal of Pediatrics and Child Health, 1998 ; 34: 263-266.

[14] BAYER J.K, HISCOCK H., HAMPTON A., WAKE M.: «Sleep problems in young infants and maternal mental and physical health », Journal of Pediatrics and Child Health, 2007; 43: 66-73.

[15] BEAUQUIER-MACCOTTA B., GOLSE B. : «L'heure du lait, l'heure du lit, l'heure du loup... Libres propos sur l'endormissement des bébés et des enfants», Spirale, Dodo, l'enfant do. Le sommeil du tout-petit, $2005 ; 34: 43-47$.

[16] BENOIT D., ZEANAH C.H., BOUCHER C., MINDE K.K.: «Sleep disorders in early childhood: association with insecure maternal attachment», Journal of the American Academy of Child and Adolescent Psychiatry, 1992; 31 (1): 86-93.

[17] BERGER M., TAMET J.Y., BLANCHON Y. : « Thérapie familiale d'un nourrisson présentant une insomnie grave », Thérapie familiale, Genève, 1983; 4 (3): 297-306.

[18] BILLIARD M., DAUVILLIERS Y.: Les troubles du sommeil. Paris, Masson, 2005.

[19] BION W.R.: Aux sources de l'expérience. Paris, PUF, 2003.

[20] BLURTON-JONESN., ROSETTIFERREIRAC., FARQUARBROWN M., MAC DONALD: «The association between perinatal factors and later night waking", Developmental Medicine and Child Neurology, 1978; 20: 42-434.

[21] BRAUNSCHWEIG D., FAIN M. La nuit, le jour. Paris, PUF, 1975.

[22] BRAZELTON T.B. : Points forts. Paris, Stock, 1994.

[23] BRISSET C., VALETTE A. : «Représentations traditionnelles et contemporaines concernant le sommeil du jeune enfant en France », Devenir, 2000; 12 (3): 65-86.

[24] CAREY W.B. : “ Night-waking and temperament in infancy», Behavioral Pediatrics, 1974 ; 84 (5) :756-758.

[25] CAREY W.B.: «Perinatal factors and later night waking», Developmental medicine and child neurology, 1979; 21: 398-399.

[26] CHALLAMEL M.J., LOUIS J. : « Les troubles du sommeil de l'enfant de moins de 6 ans: "mon enfant dort mal"», Revue médicale de la Suisse romande, 1997; 117: 745-750.

[27] CHALLAMEL M.J.: « Les insomnies du petit enfant », Enfances \& Psy: La nuit, 2000 ; 10 : 53-58.

[28] COREN S., SEARLEMAN A.: «Birth Stress and Self-Reported Sleep Difficulty», Sleep, 1985; 8 (3) : 222-226.

[29] CRAMER B., PALACIO-ESPASA F. : La pratique des psychothérapies mère-bébé: études cliniques et techniques. Paris, PUF, Le fil rouge, 1993. hypotheses exist to explain the origin of these disorders. Sleep disorders represent a very large scale of diverse emotional and conflictual troubles, from the most common to the most severe. The prognosis of these disorders relies mostly on their becoming chronic (continuance or recurrence). The long term clinical impact of insufficient sleep on cognitive development, behaviour and mood regulation is more and more studied. Treatment of infant's sleep disorders is diversified. Recommendations vary depending on cases and authors. Most often recommendations consist in mother-infant or parentinfant analytic psychotherapy, cognitive-behavioural treatment with interactiona guidance or family therapy. In exceptional cases, these may be associated with a pharmacological treatment.

\section{Keywords}

Sleep disorders.

Parent-infant interactions.

Hypotheses on the etiopathogeny. 
[30] CRAMER B. : Les troubles du sommeil chez l'enfant. Un entretien avec Bertrand Cramer. (Document vidéo) Centre National Audiovisuel en Santé Mentale, 1994.

[31] CRAMER B. : Que deviendront nos bébés? Paris, Odile Jacob, 1999.

[32] DAWS D.: Through the night: Helping Parents and Sleepless Infants. Free Association Books. 1989.

[33] DEBRÉ R., DOUMIC A. : Le sommeil de l'enfant avant 3 ans. Paris, PUF, 1969.

[34] DE LEERSNYDER H.: «Troubles du sommeil dans les maladies graves: l'accompagnement des enfants et des familles ", Médecine et enfance, 1999;311-314.

[35] DE LEERSNYDER H.: «L'endormissement du nourrisson», Enfances \& psy: La nuit, 2000; $10: 42-45$.

[36] DE LEERSNYDER H., MUNNICH A.: «Mélatonine chez l'enfant: mise au point et approches thérapeutiques», Médecine Thérapeutique Pédiatrie, 2001; 4: 1.

[37] DE LEERSNYDER H.: «Traitement par la mélatonine des troubles du sommeil de l'enfant», Archives de Pédiatrie, 2002 ; 9, Suppl 2 : 190-191.

[38] DE LEERSNYDER H. : «Les difficultés de sommeil du bébé de la naissance à l'âge de 6 mois», Spirale. Dodo, l'enfant do. Le sommeil du tout-petit, 2005 ; 34 : 165-170.

[39] DE LEERSNYDER H. : «Rythmes fondamentaux du bébé», Spirale, 2007; 4 (44): 33-38.

[40] DOLLANDER M. : « Les déterminants psychodynamiques des troubles du sommeil de l'enfant», Cliniques Méditerranéennes, 2002; 66: 193-216.

[41] DURIEUX M.P., MATOT J.P. : « A propos de l'insomnie primaire du nourrisson», Neuropsychiatrie de l'enfance et de l'adolescence, 1996; 44 (12): 617-624.

[42] ELIAS M.F., NICOLSON N.A., BORA C., JOHNSTON J. : « Sleep/wake patterns of breastfed infants in the first 2 years of life », Pediatrics, 1986; 77 (3): 322-329.

[43] FRAIBERG S.: Fantômes dans la chambre d'enfants. Version française: PUF, Fil Rouge, 1999. (Version originale anglaise 1989, Clinical Studies in Infant Mental Health- The first year of life).

[44] FRANCE K.G., HUDSON S.M.: «Management of Sleep disturbance: a review», Clinical Psychology Review, 1993; 13: 635- 647.

[45] FRANCE K.G., BLAMPIED N.M., HENDERSON J.M.T.: «Infant sleep disturbance», Current Pediatrics, 2003; 13: 241-246.

[46] FREUD S. (1895) : «Esquisse d'une psychologie scientifique», in La naissance de la psychanalyse. Lettres à Wilhelm Fliess. Notes et plans (1887-1902). Paris, PUF, 1956.

[47] FREUD S. (1900): L'interprétation des rêves, Paris, PUF, 1967.

[48] FREUD S. (1905): Trois essais sur la théorie sexuelle, Paris, Gallimard, 1987.

[49] FREUD S. (1911): «Les deux principes du cours des évènements psychiques», in Résultats, Idées, Problèmes. Paris, PUF,1991.

[50] FREUD S. (1926): Inhibition, symptôme et angoisse. Paris, PUF, 1975.

[51] GIORGIS F.G, CAMUFFO M., CASCIOLI P.: «Influenza dei disturbi precoci del sonno sullo sviluppo cognitivo». Atti dell'VIII Convegno di Neurologia Infantile, Istituto di Neuropsichiatria Infantile, 1987, Roma, Università La Sapienza, pp. 1-10.

[52] GOLSE B. : Le développement affectif et intellectuel de l'enfant. Paris, Masson, 1985.

[53] GOLSE B. «Les facteurs de risque des troubles du sommeil chez l'enfant», Intervention au $2^{\mathrm{e}}$ Colloque International sur la Prévention, Université Nancy 2. 2000.

[54] GOODLIN-JONES B.L., BURNHAM M.M., GAYLOR E.E., ANDERS T.F. : « Night-waking, sleep/wake organisation, and self-soothing in the first year of life ", Journal of Developmental and Behavioral Pediatrics, 2001 ; 22 (4): 226-233.

[55] GOVINDAMA Y.: « Troubles du sommeil du jeune enfant et thérapies associées », Neuropsychiatrie de l'enfance et de l'adolescence, 2002 ; 50: 103-113.

[56] GOVINDAMA Y.: « Trouble du sommeil chez le jeune enfant et le mode de coucher: une étude transculturelle ", L'Evolution Psychiatrique, 2004; 69: 49-65.

[57] GREEN A. : Narcissisme de vie, narcissisme de mort. Paris, Editions de Minuit, 1983. 
[58] GREGORY A.M., O'CONNOR T.G. : « Sleep problems in childhood: a longitudinal study of developmental change and association with behavioral problems", Journal of the American Academy of Child \& Adolescent Psychiatry, 2002; 41 (8): 964-971.

[59] GUEDENEY A., KREISLER L. : «Sleep Disorders in the First 18 Months of Life: Hypothesis on the Role of Mother-child Emotional Exchanges", Infant Mental Health Journal, 1987 ; 8 (3) : 307-318.

[60] GUEDENEY A. : «Les troubles de l'endormissement et du sommeil au cours des trois premiers semestres », in LEBOVICI S, WEIL-HALPERN F. (dir.): Psychopathologie du bébé, Paris, PUF, 1989 .

[61] GUEDENEY A. : «La dépression du nourrisson: nécessité d'une définition et d'une évaluation précises", Journal de pédiatrie et de puériculture, 1990 ; 7, 436-439.

[62] GUEDENEY A., ALLILAIRE J.F. : « Le concept de transgénérationnel: points de rencontre avec la théorie de l'attachement», in Interventions psychologiques en périnatalité. Paris, Masson, Coll. Médecine et Psychothérapie, 2001.

[63] HISCOCK H., WAKE M. : « Infant sleep problems and post-natal depression: a community based study», Pediatrics; 2001; 107 (6): 1317-1322.

[64] HISCOCK H., BAYER J.K., GOLD L., et al. : « Improving infant sleep and maternal mental health: a cluster randomised trial », Archives of Diseases of Childhood, 2007; 92: 952-958.

[65] HISCOCK H., BAYER J.K., HAMPTON A., et al. : « Long-term mother and child mental health effects of a population-based infantsleepintervention: cluster randomised, controlled trial», Pediatrics, 2008; 122:621-627.

[66] HOUZEL D. : « Les enjeux de la parentalité », in Collectif : La parentalité. Défi pour le troisième millénaire. Un hommage à Serge Lebovici. Paris, PUF, Le Fil Rouge, 2002.

[67] JAN J.E., FREEMAN R., FAST D.K.: « Melatonin treatment of sleep-wake cycle disorders in children and adolescents», Developmental Medicine and Child Neurology, 1999; 41: 491-500.

[68] KAHN A., GROSWASSER J., SOTTIAUX M., REBUFFAT E., et al.: «Prone or supine body position and sleep characteristics in infants», Pediatrics, 1993 ; 91 (6): 1112-5.

[69] KAHN A., MOZIN M.J., REBUFFAT E., et al. : " Intolérance au lait de vache, agitation et insomnie du nourrisson», Journal de Pédiatrie et de Puériculture, 1998; 7: 406-413.

[70] KAHN A. : Le sommeil de l'enfant, mode d'emploi pour les parents d'un petit insomniaque. Paris, Odile Jacob, 1998.

[71] KAHN A. : « Le sommeil de l'enfant et les médicaments», Enfances \& psy. La nuit, 2000 ; $10: 51-52$.

[72] KATARIA S., SWANSON M.S., TREVATHAN G.E. : «Persistence of sleep disturbances in preschool children », The Journal of Pediatrics, 1987 ; 110 (4): 642-646.

[73] KEENER A., ZEANAH C.H., ANDERS T.F.: « Infant temperament, Sleep organisation and Nighttime Parental Intervention», Pediatrics, 1988; 81: 762-771.

[74] KNAUER D., PALACIO-ESPASA F. : « Interventions précoces parents-enfants: avantages et limites», La psychiatrie de l'enfant, 2002; XLV (1): 103-132.

[75] KNAUER D., PALACIO-ESPASA F. : « Les thérapies brèves conjointes mères ou parentsbébés: évaluations, évolutions et processus cliniques», Psychothérapies, 2006 ; 26 (2): 97-102.

[76] KREISLER L., FAIN M., SOULÉ M.: L'enfant et son corps. Paris, PUF, 1974.

[77] KREISLER L. : «Désordres psychosomatiques du nourrisson: les troubles du sommeil » in LEBOVICI S, DIATKINE R, SOULÉ M.(dir.) : Nouveau traité de psychiatrie de l'enfant et de l'adolescent, Vol. 3: 2039-51. Paris, PUF,. 1985.

[78] KRIES R., KALIES H., PAPOUSEK M.: « Excessive crying beyond 3 months may herald other features of multiple regulatory problems", Archives of Pediatrics \& Adolescent Medicine, 2006 ; $160: 508-511$

[79] LAM P., HISCOCK H., WAKE M.: «Outcomes of infant sleep problems: a longitudinal study of Sleep, Behavior, and Maternal Well-Being», Pediatrics, 2003; 111: 203-207 
[80] LECENDREUX M. : «Les troubles du sommeil chez l'enfant», Enfances \& Psy, $2000 ; 10$ : 46-50

[81] LE HEUZET M.F., JACQZ-AIGRIN E. : «Les médicaments des troubles du sommeil de l'enfant», Archives of Pediatrics, 2002; 9, Suppl. 2: 187-189.

[82] LE NESTOUR A., TARNOPOLSKY D., MOURGUES M.: «Bébé Léopold: le bébé zébulon», Enfances \& Psy, $2001 ; 2$ (14) : 35-48.

[83] LE NESTOUR A., APTER-DANON G., TARNOPOLSKY D. : " Les bébés insomniaques et hyperagités", in BYDLOWSKI M. (dir.) : Des mères et leurs nouveau-nés, recherches et interventions autour de la naissance, Paris, ESF. 2002, pp. 141-162.

[84] LOZOFF B., WOLF A.W., DAVIS N.S.: «Sleep Problems Seen in Pediatric Practice», Pediatrics, $1985 ; 75: 477-483$.

[85] MAIN M., GOLDWYN R. : Adult Attachment Interview. University of California, Berkeley, 1989.

[86] MANZANO J., PALACIO-ESPASA F., ZILKHA N.: Les scénarios narcissiques de la parentalité. Clinique de la consultation thérapeutique. PUF, Fil Rouge, 1999.

[87] MARCELLI D.: Psychopathologie des conduites d'endormissement et du sommeil. Enfance et psychopathologie. $7^{e}$ édition, Masson, coll. Les âges de la vie, 2006.

[88] MARTIN J., HISCOCK H., HARDY P., DAVEY B., WAKE M.: «Adverse Associations of Infant and Child Sleep Problems and Parental Health: An Australian Population Study», Pediatrics, 2007; 119 : 947-955.

[89] MAZET P., STOLERU S.: Troubles du sommeil. Psychopathologie du nourrisson et du jeune enfant, Développement et interactions précoces. $3^{e}$ édition, Masson, coll. Les âges de lavie, 2003.

[90] MCDOUGALL J. : «The psychosoma and the psychoanalytic process», International Revue of Psycho-analysis, 1974; I: 437-459, cité par DAWS D.

[91] MENNELLA J.A, BEAUCHAMP GK. : « The transfer of alcohol to human milk. Effects on flavor and the infant's behavior», New England Journal of Medicine, 1991.

[92] MENNELLA J.A., GUERRISH C.J. : « Effects of exposure to alcohol in mother's milk on infant sleep ", Pediatrics, 1998; 101 (5): 1-5.

[93] MENNELLA J.A., GARCIA-GOMEZ P.L. : «Sleep disturbances after acute exposure to alcohol in mothers'milk», Alcohol, $2001 ; 25$ (3): 153-158.

[94] MENNELLA J.A., YOURSHAW L.M., MORGAN L.K. : «Breast feeding and smoking: Shortterm effects on infant feeding and sleep », Pediatrics, 2007; 120 (3): 497-502.

[95] MINDE K., POPIEL K. : « The Evaluation and Treatment of Sleep Disturbances in Young Children », Journal of Child Psychology and Psychiatry, 1993; 34 (4): 521-533.

[96] MINDELL J.A., KUHN B., LEWIN D.S., MELTZER L.J., SADEH A.: «Behavioral treatment of bedtime problems and night wakings in infants and young children», Sleep, 2006; 29 (10): 12631276.

[97] MISES R., FORTINEAU J., JEAMMET P., LANG J.-P., MAZET P., PLANTADE A., GUEMADA N. : « Classification Française des Troubles Mentaux de l'Enfance et de l'Adolescence (CFTMEA) », Psychiatrie de l'Enfant, 1988 ; XXXI (1), 67-134.

[98] MOORE M.S.: «Disturbed attachment in children: a factor in sleep disturbance, altered dream production and immune system dysfunction", Journal of Child Psychotherapy, 1989; 15: 1.

[99] MOORE T., UCKO L.E. : « Night Waking in early infancy», Archives of Disease in Early Childhood, 1957, $32:$ 333-342.

[100] MORGENTHALER T.I., OWENS J., ALESSI C., BOEHLECKE B., BROWN T.M., et al.: «Practice parameters for behavioral treatment of bedtime problems and night waking in infants and young children », Sleep, $2006 ; 29$ (10): 1277-81.

[101] NEUBERGER R.: «Place de la thérapie familiale dans le traitement de l'enfant», Archives de Pédiatrie, 2002; 9, Suppl. 2: 197-199.

[102] O'CONNOR T.G. : «Prenatal mood disturbance predicts sleep problems in infancy and toddlerhood», Early Human Development, 2007 ; 83: 451-458. 
[103] ORGANISATION MONDIALE DE LA SANTÉ: La CIM X. Classification Internationale des troubles mentaux et des troubles du comportement: descriptions cliniques et directives pour le diagnostic. Genève, Organisation Mondiale de la Santé, 1992.

[104] OSKARG.J.,ZINGGELER FUHRERH., OGLOWSTEINI., MOLINARIL. REMOLARGOH.: "A Longitudinal Study of Bed Sharing and Sleep Problems Among Swiss Children in the First 10 Years of Life », Pediatrics, $2005 ; 115: 233-240$.

[105] OWENS J.A., FRANCE K.G., WIGGS L.: «Behavioural and cognitive-behavioural interventions for sleep disorders in infants and children: A review», Sleep Medicine Reviews, 3: 281-302.

[106] OWENS J.A., ROSEN C.L., MINDELL J.A.: « Medication use in the treatment of pediatric insomnia: results of a survey of community-based pediatricians", Pediatrics, 2003; 111: 628-635.

[107] OWENS J.A. : «Pharmacotherapy of Pediatric Insomnia », American Academic Journal of Child and Adolescent Psychiatry, 2009 ; 48 (2): 99-107.

[108] PALIX C. : «Le sommeil à l'aube de la vie : le prématuré», Spirale, Dodo, l'enfant do. Le sommeil du tout-petit, $2005 ; 34: 71-86$.

[109] PARET I. : « Night Waking and its relationship to mother-infant interaction in nine monthold-infants», in CALL J.D., GALENSON E.G., TYSON R.L. (dir.) : Frontiers of Infant Psychiatry, New York, Basic Books, 1983, pp. 171-177.

[110] PÉLISSOlO A., LECENDREUX M., MOUREN-SIMÉONI M.C. : « Utilisation des hypnotiques chez l'enfant: description et analyse », Archives de Pédiatrie, 1999; 6: 625-630.

[111] RICHMAN N. « Depression in Mothers of Preschool Children», Journal of Child Psychology and Psychiatry, 1976; 17: 75-78.

[112] RICHMAN N.: « A community survey of characteristics of one to tow-years-old with sleep disruption", American Academic Journal of Child and Adolescent Psychiatry, 1981; 20: 281-291.

[113] RICHMAN N. : « Sleep problems in young children », Archives of Disease in Childhood, $1981 ; 56: 491-493$.

[114] RICHMAN N., DOUGLAS J., HUNT H., LANSDOWN R., LEVERE R.: «Behavioural methods in the treatment of sleep disorders : a pilot study", Journal of Child Psychology and Psychiatry, $1985 ; 26: 591-598$.

[115] RICHMAN N. : " A Double-Blind Drug Trial of Treatment in Young Children with Waking Problems», Journal of Child Psychology and Psychiatry, 1985; 26 (4): 591-598.

[116] SADEH A., LAVIE P., SCHER A., TIROSH E., EPSTEIN R. : «Actigraphic home-monitoring sleep-disturbed and control infants and young children : a new method for pediatric assessment of sleep-wake patterns », Pediatrics, 1991; 87 (4): 494-9.

[117] SADEH A., DARK I., VOHR B.R. : « Newborn sleep wake patterns : the role of maternal, delivery and infant factors», Early Human Development, 1996; 44: 113-126.

[118] SADEH A. : «Cognitive-behavioral treatment for childhood sleep disorders», Clinical Psychology Review, 2005; 25 (5): 612-628.

[119] SADEH A., FLINT-OFIR E., TIROSH T., TIKOTSKY L. : «Infant Sleep and Parental SleepRelated Cognitions», Journal of Family Psychology, 2007; 21 (1): 74-87.

[120] SCHER A., AMIR T., TIROSH E. : «Object Concept and sleep regulation », Perceptual and Motor Skills, $2000 ; 91$ : 402-404.

[121] SO K., ADAMSON T.M., HORNE R.S.: «The use of actigraphy for assessment of the development of sleep/wake patterns in infants during the firsts 12 months of life», Journal of Sleep Research, 2007; 16(2): 181-187.

[122] SPRUYT K., AITKEN R.J., SO K., CHARLTON M., ADAMSON T.M., HORNE R.S.C.: «Relationship between sleep/wake patterns, temperament and overall development in term infants over the first year of life ", Early Human Development, 2008; 84: 289-296.

[123] STOLERU S., NOTTELMANN ED., BELMONT B., RONSAVILLE D. : «Sleep Problems in Children of Affectively III Mothers», Journal of Child Psychology and Psychiatry, 1997 ; 38 (7) : 831-841. 
[124] STORK H.E.: «Bercements, berceaux, berceuses», Enfances \& psy. La nuit, 2000; 10: 28-41. [125] STORK H.E., HOSHI-WATANABE M., DONG H.P., VALETTE A. : « Le sommeil du jeune enfant et ses troubles: Une étude comparative entre trois cultures (Chine/Taiwan, France, Japon)», Neuropsychiatrie de l'Enfance et de l'Adolescence, 2000 ; 48: 70-79.

[126] SUTTER A.L., DEMOTTES-MEINARD J., HENRY C., BOURGEOIS M.: «Sommeil en période gravido-puerpérale chez la mère, le fœtus et le nouveau-né», Confrontations psychiatriques, 1997; 38: 337-356.

[127] SZWEC G. : “La mère "surintricante”», Revue Française de psychanalyse, 2002; 66 (5): 1789-1797.

[128] THUNSTRÖMM.: «Severe sleep problems in infancy associated with subsequent development of Attention-deficit/Hyperactivity disorder at 5,5 years of age ", Acta Paediatrica, 2002; $91(5): 584-592$.

[129] TOUCHETTE E., PETIT D., PAQUET J., BOIVIN M., JAPEL C., et al. : «Factors Associated With Fragmented Sleep at Night Across Early Childhood", Archives of Pediatrics \& Adolescent Medicine, 2005 ; 159: 242-249.

[130] WEISSBLUTH M. : « Sleep duration and Infant Temperament. Brief Clinical and Laboratory Observations », The Journal of Pediatrics, 1981 ; 99 (5): 817-819.

[131] WINNICOTT D.W.: De la pédiatrie à la psychanalyse. Paris, Payot, 1969.

[132] WINNICOTT D.W. : Jeu et réalité. Paris, Gallimard, 1975.

[133] WINNICOTT D.W. : " La crainte de l'effondrement », Nouvelle Revue de Psychanalyse, Gallimard, Paris.1975; 11.

[134] WOLKE D., RIZZO P., WOODS S. : « Persistent infant crying and Hyperactivity Problems in Middle Childhood», Pediatrics, $2002 ; 109 ; 1054-1060$.

[135] ZEANAH C., BORIS N.W., LARRIEU J.A. : «Infant Development and Developmental Risk: A Review of the Past 10 years", Journal of the American Academy of Child and Adolescent Psychiatry, 1997; 36: 165-178.

[136] ZERO TO THREE, NATIONAL CENTER FOR CLINICAL INFANT PROFRAMS (1994): Diagnostic Classification: 0-3. Diagnostic Classification of Mental Health and Developmental Disorders of Infancy and Early Childhood, Washington, DC. Trad. française, Genève, Médecine et Hygiène, 1998.

[137] ZERO TO THREE, NATIONAL CENTER FOR CLINICAL INFANT PROFRAMS (2005) : 2d edition revised. Diagnostic Classification: 0 - 3R. Diagnostic Classification of Mental Health and Developmental Disorders of Infancy and Early Childhood, Washington, DC. Trad. française P. Perret, Devenir, 2009 numéro special, Médecine et Hygiène, Genève.

[138] ZUCKERMAN B., STEVENSON J, BAILY V. : “ Sleep problems in early childhood: continuities, predictive factor and behavioural correlates », Pediatrics, 1987; 80 (5): 664-671. 